\title{
TESTES VISUOMOTORES EM CRIANÇAS COM ESTRABISMO
}

\author{
Jorge Alberto F. Caldeira* \\ Beatriz H. Whitaker Ferreira ** \\ MaRia CECILIA LAPA **** \\ MaRIA ANTONIA LAULETA***
}

O desenvolvimento da visão binocular nos primeiros anos de vida é um processo complexo que está na dependência de dois fatôres: o primeiro diz respeito à formação de imagens uni-oculares separadas que devem, porém, ser semelhantes, admitindo-se disparidade entre as mesmas até um certo limite; o segundo refere-se à integração das imagens em um nível mais elevado, formando-se uma impressão mental única.

Os reflexos relacionados à visão binocular podem deparar com obstáculos que a perturbarão em grau variável. Tais obstáculos pertencem a quatro categorias (Duke-Elder ${ }^{4}$ ) : ópticos, sensoriais, motores e centrais. Os obstáculos centrais são certamente causa de tropia de aparecimento precoce (Douglas ${ }^{3}$ ).

A estrabismo associado a problemas de aprendizado escolar em crianças. com paralisia cerebral tem sido objeto de numerosos estudos. Abercrombie ${ }^{1}$, estudando êsses problemas, sugere que o estrabismo pode gerar dificuldades. de ordem perceptiva em crianças com paralisia cerebral e, portanto, causar disturbios na leitura e/ou na escrita. O objetivo desta pesquisa foi comparar um grupo de crianças estrábicas, sem lesões cerebrais, com um grupo. de crianças normais, para verificar se as crianças com estrabismo apresentam maiores dificuldades na realização dos testes.

Há muitos testes psicológicos que solicitam a criança com estímulos visuais, auditivos e táteis. Foram escolhidos testes perceptivos, visuomotores e de nível mental para comparação entre os dois grupos de crianças, visando a uma inicial verificação das diferenças que poderiam estar relacionadas com o estrabismo, levantando a hipótese inicial de que crianças nestas. condições apresentam mais dificuldade nos testes visuomotores.

\section{MATERIAL E MÉTODOS}

Foram examinadas 43 crianças vindas para consulta, consecutivamente, na Seção. de Motilidade Extrínseca do Departamento de Oftalmologia e Otorrinolaringologia da Faculdade de Medicina da Universidade de São Paulo. Foram incluidos apenas

Trabalho da Disciplina de Oftalmopediatria, Departamento de Oftalmologia e Otorrinolaringologia (Professor Paulo Braga de Magalhães), Faculdade de Medicina da Universidade de São Paulo, apresentado ao XV Congresso Brasileiro de Oftalmologia (Pôrto Alegre) 27 abril-2 maio, 1969: * Professor de Disciplina de Oftalmopediatria; ** Psicóloga; *** Ortoptista. 
portadores de tropia constante ou intermitente (Tabela 1), sendo excluidos aquêles que apresentavam foria. As crianças não foram submetidas sistemàticamente a exame neurológico, porém não exibiam sintomas ou sinais de afecção do sistema nervoso. Um exame oftalmológico prévio, inclusive refração sob cicloplegia, foi seguido da correção óptica total nos casos de esotropia. Nas exotropias foi corrigida a miopia; havendo hipermetropia, apenas o componente anisometrópico da mesma foi corrigido. $O$ astigmatismo foi totalmente corrigido, tanto na esotropia como na exotropia. O exame da motilidade extrínseca só foi feito, no minimo, três semanas após o uso constante das lentes. Seguiram-se, então, os testes visuomotores.

\section{N.o de casos}

\begin{tabular}{lrrr}
\hline \hline Esotropia & 36 & $(83,7 \%)$ \\
Esotropia intermitente & 4 & $(9,3 \%)$ \\
Exotropia & 2 & $(4,7 \%)$ \\
Exotropia intermitente & 1 & $(2,3 \%)$ \\
\hline Tabela 1 - Perturbaçóses & da motili- \\
dade extrinseca nas 43 & crianças \\
& estudadas.
\end{tabular}

N.o de casos

\begin{tabular}{ll}
\hline \hline Fixação central & $32(74,4 \%)$ \\
Fixação excêntrica & $11(25,6 \%)$
\end{tabular}

rabela 2 - Fixação monocular das 43 crianças com estrabismo.

As características da fixação monocular, estudadas com o visuscópio, constam da tabela 2. Quando do exame de um ôlho, o contralateral foi sempre ocluido. Levou-se em conta o aspecto objetivo da fixação, de acôrdo com a posição da mira do visuscópio no fundo-de-ôlho. Não foi considerada, mesmo quando o paciente informava corretamente, a direção visual principal.

A idade dos 43 pacientes oscilava entre 4 e 9 anos (Tabela 3). Foi inicialmente determinado o quociente intelectual de todos os pacientes, sendo utilizado o teste de Terman e Merrill 7, forma L-M. A seguir foi aplicado o teste dos bastões de Goldstein-Scheerer, conforme apresentação de Taylor ${ }^{*}$. Em primeiro lupar foi visada a capacidade de copiar, com a criança imitando a figura armada pelo examinador. Ulteriormente foì examinada a memória: formada a figura pelo examinador, o paciente observava-a por certo tempo, sendo então desfeita para que êle a reproduzisse. Três figuras eram copiadas e 10 feitas de memória. 0 teste seguinte foi de Bender-Santucci, de acôrdo com a exposição de Zazzo, com as 6 provas preconizadas.

\begin{tabular}{ccrrr}
\hline Idade (anos) & $\begin{array}{r}\text { Grupo contrôle (44) } \\
\text { N.o de casos }\end{array}$ & $\begin{array}{c}\text { Grupo de pacientes (43) } \\
\text { N.o de casos }\end{array}$ \\
\hline 4 & 6 & $(13,6 \%)$ & 7 & $(16,3 \%)$ \\
5 & 14 & $(31,8 \%)$ & 11 & $(25,6 \%)$ \\
6 & 14 & $(31,8 \%)$ & $12(27,9 \%)$ \\
7 & 5 & $(11,4 \%)$ & 5 & $(11,6 \%)$ \\
8 & 4 & $(9,1 \%)$ & 6 & $(13,9 \%)$ \\
9 & 1 & $(2,3 \%)$ & 2 & $(4,7 \%)$ \\
\hline
\end{tabular}

Tabela 3 - Distribuição etária dos 43 pacientes e 44 contrôles.

Para o estudo da estereognosia foi aplicado o teste de Tyson, segundo Abercrombie ${ }^{1}$. Apresentava-se à criança um quadro com depressões de diferentes formatos (círculo, quadrado, elipse, semi-elipse, hexágono, losango, retângulo, triângulo, cruz, estrêla), onde poderiam ser encaixados os sólidos correspondentes. Estes eram, a seguir, oferecidos, um de cada vez, ao contacto manual, ficando, porém, fora do alcance da visão. As peças eram palpadas pela mão dominante, enquanto 
a outra apontava a depressão correspondente. A seguir foi utilizada para palpar a mão não dominante, somando-se o acêrto das duas.

Para têrmo de comparação, foi escolhido um grupo-contrôle, de 44 crianças, sem aparente tropia, mas que não foram submetidas a exame oftalmológico. Estas pertenciam, aproximadamente, aos mesmos grupos etários (Tabela 3) e ao mesmo nível sócio-econômico dos pacientes com tropia. Foram submetidas aos mesmos testes anteriormente descritos, observados os mesmos cuidados mencionados.

\begin{tabular}{|c|c|c|}
\hline $\begin{array}{l}\text { Quociente } \\
\text { intelectual }\end{array}$ & $\begin{array}{l}\text { Grupo contrôle (44) } \\
\text { N.o de casos }\end{array}$ & $\begin{array}{c}\text { Grupo de pacientes (43) } \\
\text { N.o de casos }\end{array}$ \\
\hline de $\quad 50$ a 79 & $(6,8 \%)$ & $9 \quad(20,9 \%)$ \\
\hline $\begin{array}{llll}\text { de } & 80 & \text { a } & 89\end{array}$ & $10 \quad(22,7 \%)$ & $14(32,6 \%)$ \\
\hline de 90 a 110 & $28 \quad(63,7 \%)$ & $19(44,2 \%)$ \\
\hline acima de 110 & $3 \quad(6,8 \%)$ & $(2,3 \%)$ \\
\hline
\end{tabular}

Tabela 4 - Resultados do teste de Terman e Merrill para quociente intelectual nas 44 crianças do grupo contrôle $e$ nas 43 crianças com estrabismo.

\section{RESULTA DOS}

O quociente intelectual (Q.I.), verificado pelo teste de Terman e Merrill dos pacientes e das crianças do grupo de contrôle figura na tabela 4. As crianças estrábicas com Q.I. entre 50 e 79 foram $9(20,9 \%)$; 14 obtiveram o Q.I. entre 80 e 89 $(32,6 \%)$. Portanto, entre as crianças com perturbação da motilidade extrinseca - nivel intelectual foi abaixo da média em 53,5\%, enquanto que no grupo de criancas normais apenas 29,5 tinham Q.I. abaixo de 90 , das quais $6,8 \%$ com Q.I. entre 50 e 79. Elegendo como valor arbitrário o Q.I. 85, verifica-se (Tabela 5) que $39,5 \%$ dos pacientes situavam-se abaixo daquela cifra, enquanto $18,2 \%$ dos contrôles o atingiam.

Quanto ao teste de Goldstein-Scheerer, foi constatada média inferior em $39,5 \%$ dos pacientes e em $18,2 \%$ dos contrôles (Tabela 5); os èrros considerados foram de "distorção" das figuras copiadas e feitas de memória. No teste de BenderSantucci a média inferior fol encontrada em $48,8 \%$ dos pacientes e em $45,4 \%$ dos contrôles (Tabela 5).

O exame de lateralidade revelou ser a mesma homogênea em 53,5\% dos pacientes e $47,7 \%$ dos contrôles; a lateralidade cruzada alcançou as cifras, respectivamente, de $44,2 \%$ e $43,2 \%$; para a lateralidade indefinida elas foram de $2,3 \%$ e $9,1 \%$. (Tabela 5).

O teste de estereognosia de Tyson revelou média inferior em $44,0 \%$ dos pacientes e em $38,6 \%$ dos contrôles (Tabela 5 ).

\section{COMENTARIOS}

Entre os pacientes utilizados na presente pesquisa a incidência de esotropia predominou largamente, como acontece normalmente em crianças estrábicas. De outra parte, a incidência de fixação excêntrica foi alta $(25,6 \%)$. Sendo tôdas as crianças de uma condição sócio-econômica relativamente baixa, em geral foram encaminhadas para tratamento especializado depois de decorrido tempo mais ou menos longo após o aparecimento de tropia, o que explica a perturbação da fixação monocular. 
Se se considerar o Q.I. (Tabelas 4 e 5), verifica-se ser o mesmo nitidamente inferior nos pacientes, em relação às crianças do grupo de contrôle. O Q.I. das crianças estrábicas revelou-se $44 \%$ dentro da média enquanto que nas crianças normais foi de $63 \%$. O nível mental foi abaixo de $89 \mathrm{em}$ $28 \%$ das crianças normais e em $52 \%$ das crianças estrábicas. Esta verificação faz supor a existência de dificuldades ligadas às perturbações da motilidade ocular extrínseca, que devem ter prejudicado a boa realização dos testes de inteligência onde há ítens que envolvem performance visuomotora. $\dot{E}$ possivel, também, que tenha ocorrido falta de solicitação nas crianças estrábicas em virtude mesmo do problema ocular que apresentam. Seria interessante pesquisar um grupo etário mais numeroso de crianças com 7 anos, que já estivessem recebendo solicitação escolar. Neste caso poder-se-ia analisar com segurança a influência da solicitação das atividades visuomotoras, comparando com as dificuldades do aprendizado da escrita e leitura. As crianças objeto desta pesquisa, em ambos os grupos, pertenciam a uma classe sócio-econômica baixa, na qual as crianças são encaminhadas à escola depois dos 6 anos; só havia, acima de 6 anos, 13 crianças estrábicas e 10 normais. A comparação em número tão pequeno de casos não seria significativa.

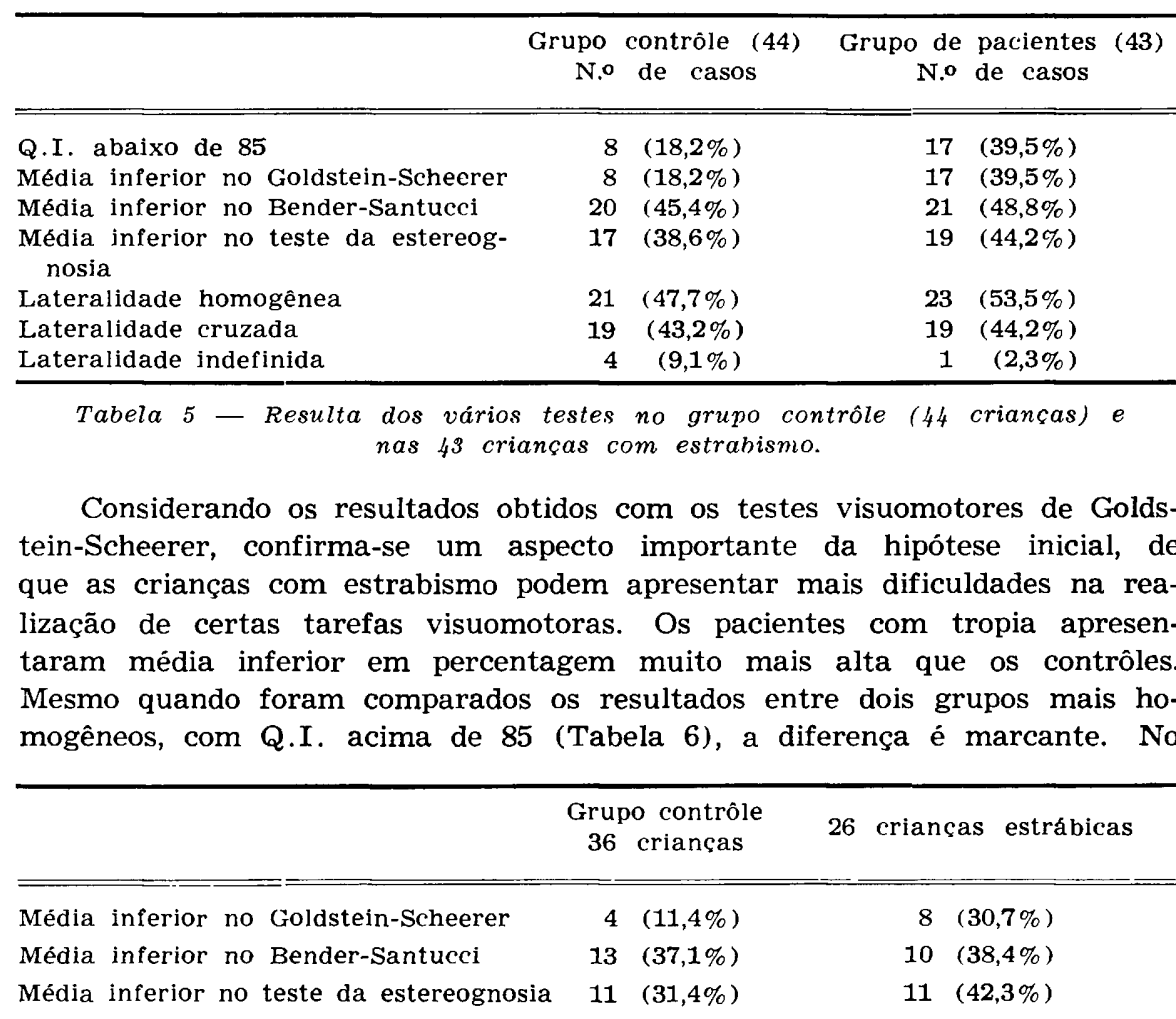

Tabela 6 - Estudo comparativo dos resultados obtidos em crianças com quociente intelectual acima de 85, tanto estrábicas (26) como do grupo-contrôle(36). 
teste de Bender-Santucci, entretanto, a diferença entre os dois grupos não foi significativa, mesmo considerando apenas aquêles com Q.I. acima de 85 . Como o trabalho com bastões implica na percepção de outra dimensão pois os desenhos fazem relêvo na superfície da mesa, talvez a tarefa seja de realização mais complexa para as crianças estrábicas. Outra hipótese para explicar a discrepância entre os resultados dos dois testes, que envolvem compreensão das relações espaciais, seria baseada no fato de haver utilização da memória visual na performance dos bastões, cujas figuras eram mostradas apenas durante algum tempo, enquanto que no teste de figuras com lápis e papel de Bender-Santucci, não há necessidade de recorrer à memória visual pois há cópia simples do modêlo, que permanece na frente da criança todo o tempo. Para comprovação destas hipóteses teria sido interessante comparar o teste de Goldstein-Scheerer e o "visual retention test" de Benton 2, nos quais as figuras são observadas por alguns segundos e depois feitas de memória, sendo que no último, como no teste de Bender-Santucci não há figuras com terceira dimensão.

Os dados colhidos nos dois grupos com a aplicação do teste de organização gráfico-perceptiva de Bender-Santucci são dificilmente avaliáveis, em virtude de terem sido usados como têrmo de comparação os resultados da adaptação francesa. A média inferior no grupo normal pode significar que os resultados colhidos com crianças francesas não se adaptam ao meio brasileiro em virtude de fatôres culturais. As nossas crianças de nível sócioeconômico baixo não recebem solicitação de ordem visuomotora e perceptiva a não ser a partir dos 7 anos, idade em que iniciam o aprendizado escolar.

A percentagem maior de média inferior obtida pelos pacientes no teste de estereognosia sugere que entre os estrábicos é maior a incidência de perturbação manual, tal como foi observado em tôdas as formas de paralisia cerebral (Monfraix e col. ${ }^{5}$ ).

Os resultados quanto à lateralidade revelam que a homogênea foi um pouco mais observada entre os estrábicos do que entre os contrôles, enquanto que a incidência da dominância cruzada foi pràticamente a mesma nos dois grupos. A lateralidade indefinida foi mais encontrada entre os contrôles.

\section{R E S U M O}

Em 43 crianças estrábicas nas quais foi determinado o quociente intelectual foram aplicados os testes visuomotores de Goldstein-Scheerer, Bender-Santucci, lateralidade e estereognosia. Os resultados foram comparados com os obtidos em 44 contrôles dos mesmos grupos etários e sócio-econômicamente semelhantes.

Sugere-se o prosseguimento desta pesquisa com o estudo comparativo entre o teste de Goldstein-Scheerer e o "visual retention test" de Benton (1963) nos quais a análise do fator memória visual pode ser útil para averiguar dificuldades relacionadas com a prontidão para a alfabetização. Sugere-se, também, o estudo de um grupo de crianças em idade escolar com e sem perturbações da motilidade ocular extrínseca para avaliar a influência da escolaridade nos resultados dos testes. 


\section{S U M M A R Y}

Visuomotor tests in children with strabismus

Forty-three children with different forms of strabismus and 44 normal children were submited to the following tests: Terman-Merrill intelligence scale, form L-M; Goldstein-Scheerer stick test; Bender-Santucci graphic perceptive organisation; Zazzo lateral dominance test; Tyson cross-modality matching test. Children with squint exhibited poorer results with the Terman-Merrill, Goldstein-Scheerer and Tyson tests as compared with normal children. The differences found with the other tests were not significant.

\section{REFERENCIAS}

1. ABERCROMBIE, M. L. J. - Perceptual Visuomotor Disorders in Cerebral Palsy. The Spastics Society \& William Heinemann Medical Books Ltd., London, 1964.

2. BENTON, A. L. - The Revised Visual Retention Test. 3rd edition. The Psychological Corporation, New York, 1963.

3. DOUGLAS, A. - The role of brain damage in the aetiology of squint. In Smith, V. H. ed. - Visual Disorders and Cerebral Palsy. The National Society \& William Heinemann Medical Books Ltd., London, 1963.

4. DUKE-ELDER, W. S. - Text-book of Ophthalmology. Henry Kimpton, London, 1949, vol. 4 .

5. MONFRAIX, C.; TARDIEU, G. \& TARDIEU, C. - Disturbances of manual perception in children with cerebral palsy. Cerebral Palsy Bull. 3:544, 1961.

6. TAYLOR, E. M. - Psychological Appraisal of Children with Cerebral Defects. Harvard University Press, Cambridge, 1959.

7. TERMAN, L. M. \& MERRILL, M. A. - Stanford-Binet Intelligence Scale. Houghton Mifflin Co., Boston, 1962.

8. ZAZzo, R. - Manual para el Examen Psicológico del Niño. Editorial Kapelusz, Buenos Aires, 1963.

Clinica Oftalmológica - Faculdade de Medicina, Universidade de São Paulo Caixa Postal 8091 - São Paulo, SP - Brasil. 